\title{
Using Universal Content Identifier (UCI) as Uniform Resource Names (URN)
}

Status of This Memo

This memo provides information for the Internet community. It does not specify an Internet standard of any kind. Distribution of this memo is unlimited.

Copyright Notice

Copyright (C) The Internet Society (2005).

Abstract

This document describes a Uniform Resource Name (URN) namespace for the National Computerization Agency (NCA) for naming persistent digital resources such as music, videos, texts, images, e-books, and other types of digital resources produced or managed by NCA.

1. Introduction

NCA is a non-profit organization with a mandate to develop and promote information infrastructure and manage public digital contents in Korea and possibly worldwide.

NCA has been supervising digitalization projects with various organizations. The contents involved in such projects include millions of technical papers, cultural properties, educational materials, scientific research reports. Those organizations manage the digital contents in a standardized way set by NCA and provide users with various content services via internet.

NCA wishes to issue globally unique and persistent IDs to digital resources and assign relevant organizations as its registration agencies under the Universal content Identifier (UCI) scheme. It is beneficial for $\mathrm{NCA}$, its registration agencies, and the value chain players in e-commerce, to have the UCI URN namespace, under which all the contents and components produced and managed by NCA are digitally registered, identified, and resolved.

NCA has been developing and operating the UCI system that provides registration, resolution, search, and administration functionalities. 
The functionalities are going to be added and modified once the use of UCI URN namespace is approved. NCA would like to assign unique, permanent, and location-independent names based on the URNs for the resources it produces or manages. See http://www.nca.or.kr/eindex.htm for more information on NCA.

This namespace specification is for a formal namespace.

2. Specification Template

Namespace ID:

"UCI"

Registration Information:

Registration Version Number: 1 Registration Date: 2004-07-xx

Declared registrant of the namespace:

Name : Sang-ug Kang

Affiliation: National Computerization Agency

Address : NCA Bldg. 77 Mookyo-Dong, Chung-Ku, Seoul, Korea

Phone : +82 (2) 2131-0443

Email : <sukang@nca.or.kr>

Web : <http://www.nca.or.kr> or <http://home.uci.or.kr>

Declaration of syntactic structure:

The Namespace specific string of all URNs assigned by NCA conforms to the syntax defined in section 2.2. of RFC2141, "URN Syntax"[1]. The syntax convention is described in the form of ABNF rules [2] as the following.

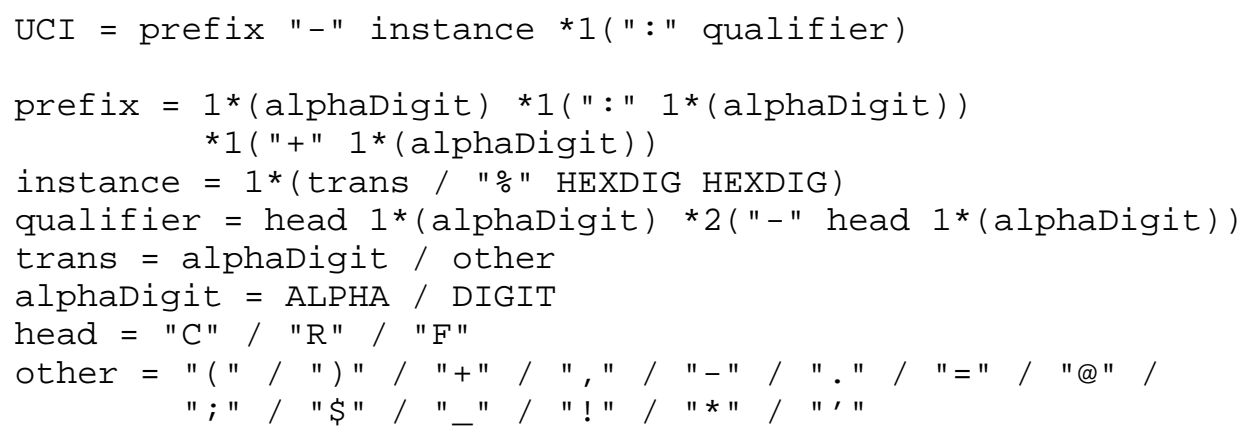


The UCI identifier consists of two parts: prefix code and content code. The content code is also divided into instance code and qualifier code, which is optional. A prefix code is given to a registration agency, subordinate registration agency, and/or its registrants such as 'G3000+music', 'I600', 'I500+paper', and so forth. A content code is issued to each individual digital resource such as 'cii90007',' '8987409', and so on.

Relevant ancillary documentation:

None.

Identifier uniqueness considerations:

It is the responsibility of the registration authority, or NCA, to guarantee the uniqueness of identifiers and the names of subordinate naming authorities. For example, a software tool developed and used by NCA checks if a UCI identifier is being reassigned and verifies that it is assigned to at most one resource during the registration process.

Identifier persistence considerations:

Registration Agencies are all eligible to maintain the usability of the UCI URNs for a reasonably long period. As a government sponsored organization, NCA will operate a backup service and make an effort to find a substitute in case a registration agency becomes out of operation.

Process of identifier assignment:

Assignment of UCI identifier is delegated to the registration agencies. NCA appoints UCI registration agencies and provides its namespace by assigning a unique registration agency code that is a part of NSS. Followed by the authorized namespace, each registration agency sets its own identifier scheme that conforms to the UCI syntactic structure.

A UCI for a digital resource is issued by the registration agency, upon a request of a registrant. The following is an example identifier.

e.g., urn:uci:I700-2987098

where, I700 is a registration agency code and 2987098 is an identifier assigned to a digital content. 
Process for identifier resolution:

Resolution is controlled by NCA and its delegatees. The list of UCI registration agency codes is maintained by the registration authority. Each registration agency maintains the list of subordinate registrant codes and identifiers registered to the registration agency.

Identifier resolution steps are as follows.

- (Stepl) Global resolution: NCA resolves which RA is dealing with the associated resource by parsing the registration agency code of the UCI.

- (Step2) Local resolution: The corresponding RA parses and resolves the rest of the identifier

The global resolution is performed by UCI resolution server that is open to public. (Currently http://rootadmin.uci.or.kr:9000/ \{UCI identifier\} will give you resolution results.)

Rules for Lexical Equivalence:

The "prefix" is case-insensitive. So there will not be two registration agencies whose names differ only in case.

urn:uci: $\{$ prefix code $\}$ :

is case-insensitive for matches. Remainder of the identifier is case-sensitive.

Conformance with URN Syntax:

The UCI syntax fully conforms to RFC2141 syntax rules for both NSS and NID.

Validation mechanism:

Valid list of prefix code will be available via both on-line and off-line upon request. The validity of the rest of identifier can be offered via on-line service. (Currently at http://rootadmin. uci.or.kr:9000/\{UCI identifier\} by typing a query UCI identifier)

Scope :

Global. 
3. Security Considerations

There are no additional security considerations beyond those normally associated with the use and resolution of URNs in general.

4. Namespace Considerations

The Korean government has been funding the UCI project with the vision that it will be an essential component in realizing interoperability of digital contents owned by individual institutes and organizations. The resolution service provided by NCA and its registration agencies via networks will play a central role in achieving such a goal. Moreover, the resolution service is open to the public without discrimination.

The assignment and use of identifiers is performed according to the rules established by NCA. It abides by the URN requirements and syntax. Within a UCI namespace, a registration agency manages subordinate namespaces and maintains the metadata of digital contents.

For making the service available to the public, a global resolution service is run by NCA through a service server and local resolution service is provided by the corresponding registration agency.

\section{Community Considerations}

The assignment and use of identifiers within the namespace are open, and the related rule is established by NCA. Registration agencies are evaluated and selected fairly and shall have the responsibility of processing registrants' requests for registering digital contents. Registration authority and registration agencies shall operate resolution servers for UCI namespace and subordinate namespaces, respectively. Digital content users can access the resolution servers to fetch resolution results. The reference software is developed, and resolution servers are now in operation.

6. IANA Considerations

The IANA has registered the "UCI" namespace in the IANA registry of URN NIDs. For more information about URN namespace, see [3] . 
7. Normative References

[1] Moats, R., "URN Syntax", RFC 2141, May 1997.

[2] Crocker, D. and P. Overell, "Augmented BNF for Syntax Specifications: ABNF", RFC 2234, November 1997.

[3] Daigle, L., van Gulik, D., Iannella, R., and P. Faltstrom, "Uniform Resource Names (URN) Namespace Definition Mechanisms", BCP 66, RFC 3406, October 2002 .

Author's Address

Sang-ug Kang

National Computerization Agency

Bldg. 77 Mookyo-Dong,

Joong-Ku, Seoul,

Korea

Phone: 82-2-2131-0443

EMail: sukang@nca.or.kr 
Full Copyright statement

Copyright (C) The Internet Society (2005).

This document is subject to the rights, licenses and restrictions contained in BCP 78, and except as set forth therein, the authors retain all their rights.

This document and the information contained herein are provided on an "AS IS" basis and THE CONTRIBUTOR, THE ORGANIZATION HE/SHE REPRESENTS OR IS SPONSORED BY (IF ANY), THE INTERNET SOCIETY AND THE INTERNET ENGINEERING TASK FORCE DISCLAIM ALL WARRANTIES, EXPRESS OR IMPLIED, INCLUDING BUT NOT LIMITED TO ANY WARRANTY THAT THE USE OF THE INFORMATION HEREIN WILL NOT INFRINGE ANY RIGHTS OR ANY IMPLIED WARRANTIES OF MERCHANTABILITY OR FITNESS FOR A PARTICULAR PURPOSE.

Intellectual Property

The IETF takes no position regarding the validity or scope of any Intellectual Property Rights or other rights that might be claimed to pertain to the implementation or use of the technology described in this document or the extent to which any license under such rights might or might not be available; nor does it represent that it has made any independent effort to identify any such rights. Information on the procedures with respect to rights in RFC documents can be found in BCP 78 and BCP 79 .

Copies of IPR disclosures made to the IETF Secretariat and any assurances of licenses to be made available, or the result of an attempt made to obtain a general license or permission for the use of such proprietary rights by implementers or users of this specification can be obtained from the IETF on-line IPR repository at http://www.ietf.org/ipr.

The IETF invites any interested party to bring to its attention any copyrights, patents or patent applications, or other proprietary rights that may cover technology that may be required to implement this standard. Please address the information to the IETF at ietfipreietf.org.

Acknowledgement

Funding for the RFC Editor function is currently provided by the Internet Society. 